\title{
Curtain falls on Britain's nuclear structure facility
}

London. A meeting earlier this month at Daresbury in Cheshire to celebrate the achievements of the Nuclear Structure Facility (NSF) was also an occasion to mourn its premature death. On Monday (29 March), the last ions will be accelerated down the 70metre high Van de Graaff accelerator to smash into nuclei at the base of the NSF, which fell victim to a financial crisis two the ion-beam crystallography work. This so-called medium-energy ion-scattering facility (MEIS) will carry out structural investigations of the surfaces and subsurface regions of materials.

Daresbury officials claim that MEIS will be "equal to the best facilities elsewhere in the world". But physicists working on the NSF point out that its predecessor has been a world leader. "The 'me-too' ar-

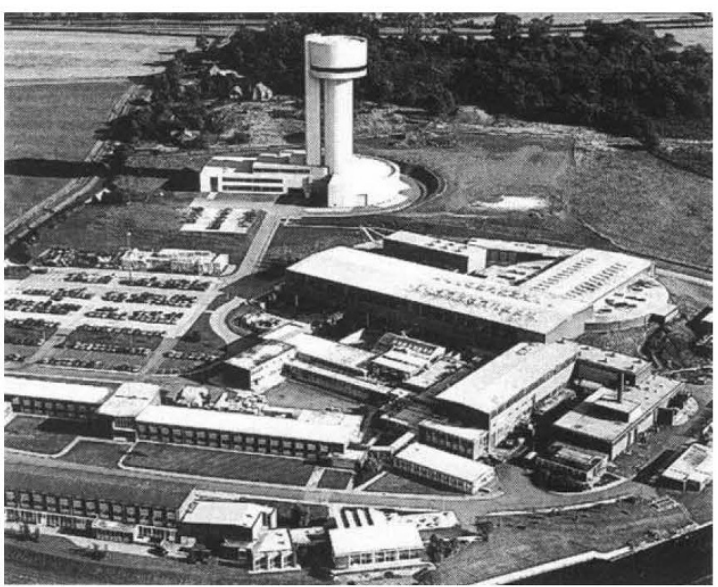

Landmark tower outlives its usefulness.

years ago at its funding agency, the Science and Engineering Research Council (SERC).

For about 25 members of the NSF staff, next week's final run will mean early retirement from a machine opened in the mid1970 s that had been expected to operate until late in the decade. For a fortunate few, a new ion-beam test facility at the Rutherford Appleton Laboratory (see right) or other projects at Daresbury will offer continuing employment.

Last year, proposals were submitted to the SERC for three projects to use the skills and equipment made available by the closure of the NSF. One was a facility to study small clusters of atoms or molecules, either in gases or attached to surfaces. A second was to build a much smaller Van de Graaff machine, capable of reaching energies up to $8 \mathrm{KeV}$, with potential applications as a mass spectrometer in fields ranging from archaeology to medicine. The third was to use the ion-beam injector to carry out crystallography studies.

Only the last has been successful. SERC committees decided that a central facility was not needed for the cluster project and that such work should remain in university departments. The proposal for an accelerator mass spectrometer was an interdisciplinary effort that fell between the responsibilities of the different research councils Some scientific respectability has been salvaged by the SERC's decision to support gument seems somewhat feeble", says John Sharpey-Shafer, who believes that closing NSF was "scandalous".

Even more jobs would have been lost if the NSF's demise had not coincided with rationalization at the nearby ICI Materials Research Centre. As part of this process, ICI has agreed to transfer to Daresbury a high-resolution Xray photoelectron spectrometer, which carries out studies of the surface of materials at the molecular level.

The machine, unique in Britain, will become the focus of a new group known as the Research Unit in Surfaces, Transforms and Interfaces (RUSTI). About ten scientists and technical staff from the NSF will be transferred to the new unit; one-third of the available time will be used by ICI and twothirds will go to SERC grant-holders and to British companies investigating new ways of processing advanced materials. "This machine fits a niche between pure research and more industrially oriented research," says Hywel Price, the current director of the NSF who will take over as director of the new research unit.

The future of the tower housing the NSF is still under discussion. The highly visible building met with such local opposition at first that the SERC agreed to pull it down at the end of the NSF's working life; it is now something of a local landmark, and the destruction order has been lifted. Mercury, a telecommunications company, plans to mount equipment on it for receiving and transmitting radio signals, and the SERC is thinking about turning it into a science visitor centre that, among other things, would try to interest young people in careers in science and technology.

The irony has not been lost on the scientists working on the NSF, who are trying to do as much research as possible before the ion beam is shut off next week. "The closure of the NSF marks the end of an era for both Daresbury and for nuclear structure research in the UK", says laboratory director Alan Leadbetter

David Dickson
Life after death for nuclear physics

London. Nuclear structure physics in Britain may be down (see left), but it is far from out. Last month, the Science and Engineering Research Council approved $£ 1.7$ million (US\$2.5 million) for a new ion-source test-bed facility to be attached to the spallation neutron source ISIS at the council's Rutherford Appleton Laboratory. British physicists view it as the first step in a campaign to secure a radioactive nuclear beam facility, Europe's next major tool for research into nuclear structure.

At present, the amount of information obtained by machines designed to study the structure of atomic nuclei such as the National Structure Facility (NSF) at Daresbury - is limited by the need to confine attention to naturally occurring nuclei. Studies are now planned at the European Laboratory for Particle Physics (CERN) of ways to add a 'post-accelerator' to the isotope separator ISOLDE in order to produce a beam of unstable radioactive ions, although still at relatively low energies. The experiments at the Rutherford Appleton Laboratory, to which a group of scientists from the NSF will now be attached, will complement these efforts by looking at techniques for producing proton beams at much higher energies and targets to withstand the intensities involved.

Using a spallation source to produce the radioactive ions may not turn out to be the best way forward; another approach is being explored at the heavy-ion accelerator GANIL in France. But success would give Britain a leg up in the competition for a machine, costing roughly $£ 50$ million, that has recently been given top priority by the European coordinating committee for nuclear physics, NuEPP. (The US Department of Energy has already asked for proposals from its laboratories for a similar machine.)

"The closure [of the NSF] was an act of folly and vandalism, but it still leaves our long-term future intact", says William Gelletly, formerly head of the NSF and now professor of physics at the University of Surrey. Gelletly is urging the SERC to persuade its European partners to locate the new machine in Britain as a way of regaining a central place in nuclear structure research that is being lost by the closure of the NSF. 